\title{
Performance Improvement by Sorting the Transform Coefficients of Host and Watermark using Unitary Orthogonal Transforms Haar, Walsh and DCT
}

\author{
H. B. Kekre ${ }^{1}$, Tanuja Sarode ${ }^{2}$, Shachi Natu ${ }^{3}$ \\ ${ }^{1}$ Senior Professor, MPSTME, NMIMS University Mumbai, ${ }^{2}$ Assosiate Professor, TSEC, Mumbai \\ University, Mumbai, ${ }^{3} \mathrm{Ph}$. D. Research Scholar, NMIMS University, Mumbai; \\ hbkekre@yahoo.com, tanuja_0123@yahoo.com, shachi_natu@yahoo.com
}

\begin{abstract}
This paper proposes a new watermarking technique in which host and watermark image is converted to frequency domain using orthogonal transforms Haar/Walsh/DCT. These transforms are applied fully, row wise and column wise to host and watermark. Transform coefficients of host image are sorted in descending order of energy and replaced by sorted transform coefficients of watermark. Column and row transforms of Haar, Walsh and DCT prove better over full transform. Further, sorting results in better imperceptibility as it minimizes the energy difference between original host's transform coefficients and replaced watermark coefficients. Proposed technique gives better performance than unsorted transform coefficients for watermarking. In this paper results of only Haar transform are given due to space limitation.
\end{abstract}

Keywords: Watermarking, column transform, row transform, cropping, binary distributed run length noise, Gaussian distributed run length noise, resizing.

\section{Introduction}

Reproduction of digital contents has become easier due to various tools available for it. At the same time demand for transmission of digital contents is growing rapidly. This makes it compulsory to have protection of data in terms of prevention from illegal copying or distribution. Watermarking serves the purpose for the same.

Watermarking refers to insertion of signature of owner of digital contents into those contents itself. It should satisfy two major constraints. First is invisibility (imperceptibility) which means original digital contents and contents after insertion of watermark should look same to the Human Visual System. Second is robustness against various attacks which try to 
destroy or remove the watermark [1]. Insertion of watermark can be done in two different domains namely spatial and frequency domain.

- Spatial domain watermarking:

Watermarking in spatial domain refers to modification to the pixel values of specific region of an image [2]. Apart from the advantage of simplicity, the major drawback of spatial domain watermarking techniques is its poor capability to embed data and less robustness to attacks. LSB technique is widely appreciated spatial domain technique. Various other spatial domain techniques have been proposed in $[1,2,3,4]$.

- Frequency domain watermarking:

Watermarking in frequency domain refers to transforming host image into frequency domain and modifying transform coefficients by watermark [3]. By applying inverse transform watermarked image is obtained. Image can be transformed into frequency domain using transforms like Discrete Cosine Transform (DCT), Discrete Fourier Transform (DFT), and Discrete Wavelet Transform (DWT) etc. Multiple watermarks can also be embedded in different frequency locations of host image to provide more robustness.

A DCT based watermarking technique is given by W. Lu et. al in [5] where watermark is embedded into the DCT coefficients of sub images which are obtained by subsampling the original image. In [4], Riaz et. al proposed a frequency domain watermarking in which encrypted data is inserted into the image. Extraction is usually the reverse procedure of insertion. Circular watermarking by amplitude modulation is proposed by Licks in [6]. The best known technique of watermarking is proposed by Cox [7] by applying DCT to the entire image. Wavelet based watermarking is proposed by Woo et. al in [8] and by Giakoumaki in [9]. Kekre, Sarode and Shachi natu have proposed DCT wavelet, Walsh wavelet and hybrid wavelet based watermarking techniques in [10-18].

In this paper, a frequency domain blind watermarking technique for digital images is proposed. It uses Haar, Walsh and Discrete Cosine transform. These transforms are applied to host images as well as to watermark to be embedded. Along with the full transform, row transform and column transforms are also used using Haar, Walsh and DCT. Transformed watermark elements are sorted and are inserted in sorted middle frequency elements of transformed host image. Varying embedding energy of watermark is used to test the robustness against various attacks by keeping in mind energy conservation property of transforms. Organization of paper is as follows. Section 2 describes proposed method. Section 3 describes results and observations for the proposed method and compares proposed work with our previous work in which watermark is inserted in frequency domain without sorting the frequency coefficients. Section 4 concludes the paper. 


\section{Proposed method}

In proposed method orthogonal transforms DCT, Walsh and Haar are explored in full, column and row version along with the concept of sorting elements of middle frequency band of host and frequency elements of watermark.

Transformed image obtained after applying full transform to it is given by

$$
\mathrm{F}=\mathrm{T}^{*} \mathbf{f}^{*} \mathrm{~T}^{\mathrm{t}}
$$

Where, $T$ is unitary, orthogonal transform matrix, $T^{\prime}$ is its transpose, $f$ is image to be transformed and $\mathrm{F}$ is transformed image. Original image can be obtained from transformed image as

$$
\mathbf{f}=\mathbf{T}^{\mathrm{t}} * \mathrm{~F}^{*} \mathbf{T}
$$

For column transform, transformed image is obtained by premultiplying image with transform matrix as shown in equation (3) and original image is obtained by premultiplying transformed image with transpose of transform matrix as shown in equation (4).

$$
\begin{aligned}
& F=T^{*} \mathbf{f} \\
& \mathbf{f}=T^{\mathrm{t}} * \mathbf{F}
\end{aligned}
$$

Row transform of an image is given by operating transposed transform matrix on rows of an image and image in spatial domain is obtained by operating transform matrix on rows of transformed image as shown in equation (5) and (6).

$$
\begin{aligned}
& F=f^{*} T^{t} \\
& f=F * T
\end{aligned}
$$

We have explored various orthogonal transforms for embedding watermark without sorting transform coefficients. It was found that Haar column transform is strongly robust against various attacks like cropping, noise addition, compression, resizing etc. also it was closely followed by Haar row transform and then by column and row versions of Walsh and DCT. Hence in this paper emphasis is on checking the performance of these transforms against various attacks when host and watermark frequency coefficients are sorted based on energy. Figure 1

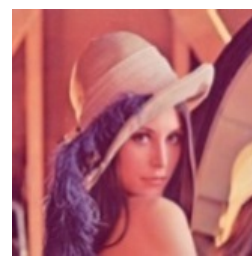

(a) Lena

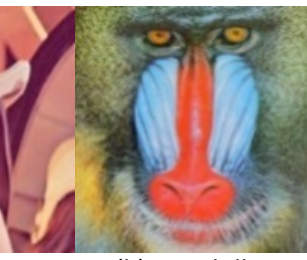

(b) Mandrill

(c) Peppers

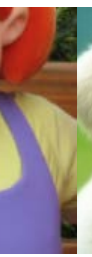

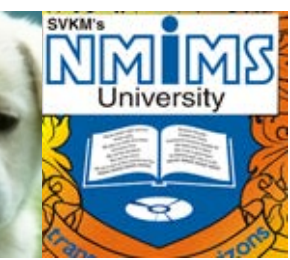

(f) NMIMS

Figure1: Host images and a watermark image used for experimental work 


\subsection{Embedding Process:}

1. Read host image and watermark image.

2. Take Haar/Walsh/Discrete Cosine Transform (full/ column/ row) of them.

3. For full transform select $\mathrm{HL}$ and $\mathrm{LH}$ band to embed the watermark. For column transform middle rows and for row transform middle columns are selected for embedding watermark.

4. Elements of watermark as well as elements from the frequency band of host are sorted in descending energy order.

5. Sorted frequency elements are normalized and scaled using suitable scaling factor such that watermark energy is $60 \%, 100 \%$ and $140 \%$ of the mid-frequency band energy of host.

6. Mid-frequency coefficients of host are replaced by scaled and sorted frequency elements of watermark.

7. Inverse transform is taken to obtain watermarked image.

\subsection{Extraction process:}

1. Read watermarked image.

2. Apply Haar/ Walsh/ DCT (full/ column/ row) to it.

3. Sort middle frequency elements of transformed watermarked image.

4. From these middle frequency elements recover frequency elements of watermark. these elements are descaled, denormalized and placed in their respective places after inverse sorting.

5. Reconstruct watermark from recovered elements by taking inverse transform.

Sorting of host and watermark frequency elements leads to minimization of energy difference between original and replaced elements and thus reduces the error between host and watermarked image.

\section{Results of proposed method against various attacks}

Results of proposed method are shown in terms of recovered watermark from attacked image and they are compared to results of watermarking without energy sorting of frequency elements of host and watermark images. Due to limitation of space results of only Haar transform for Lena image are shown.

Figure 2(a) and (b) show watermarked image Lena using full transform $\mathrm{HL}$ band and recovered watermark from it obtained using proposed method with sorting and unsorted coefficients. 


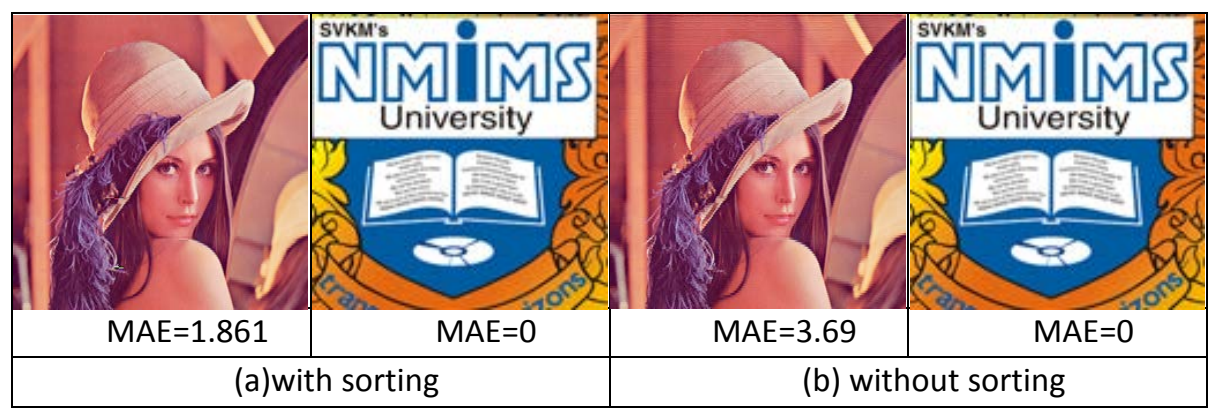

Figure 2: Watermarked Lena image and recovered watermark from it with and without sorting using HL band to embed the watermark

From Figure 2 we can see that MAE between host image and watermarked image has been reduced resulting in better imperceptibility. Figure 3 shows the similar results when LH band is used to embed the watermark. Here also, using sorted transform coefficients results in better imperceptibility.

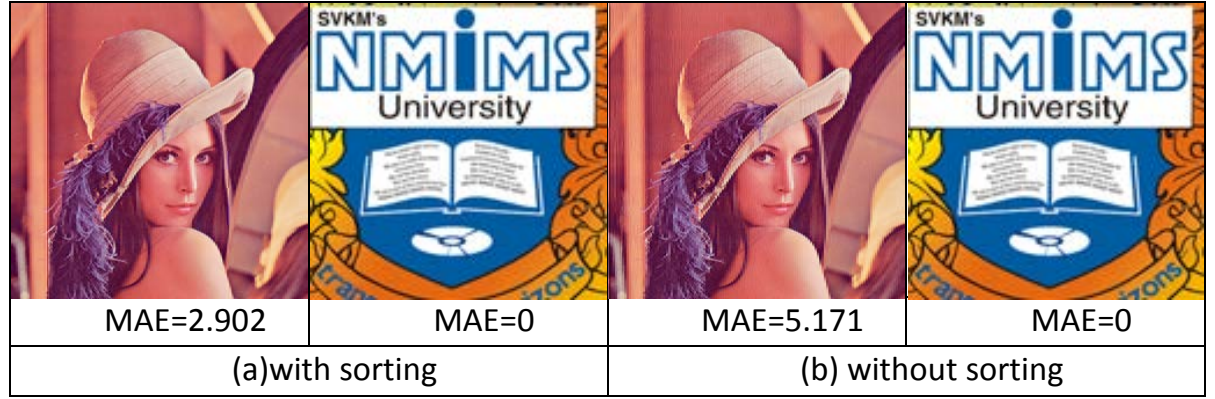

Figure 3: Watermarked Lena image and recovered watermark from it with and without sorting using LH band to embed the watermark

Figure 4 (a) shows watermarked image and recovered watermark from it using column Haar transform when sorting is performed on transform coefficients and Figure 4(b) shows the result obtained without sorting. Figure 5(a) shows watermarked image and recovered watermark from it when row Haar transform is used and transform coefficients are sorted. Figure 5(b) shows corresponding images for unsorted transform coefficients.

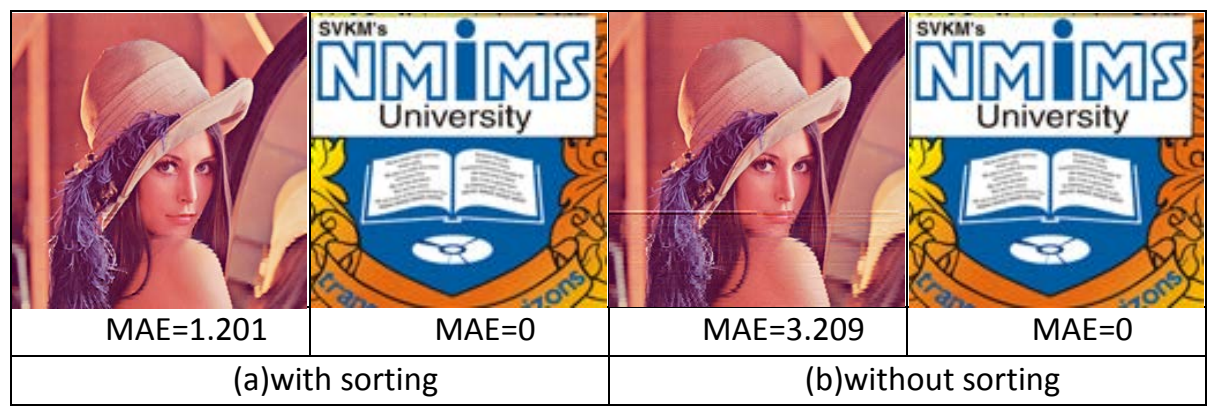

Figure 4: Watermarked image and recovered watermark from it using column Haar transform 




Figure 5: Watermarked image and recovered watermark from it using row Haar transform

From Figure 4 and Figure 5 it is very clear that sorting improves the imperceptibility of watermarked image.

\subsection{Performance evaluation of proposed method against various attacks:}

From Figure 2- Figure 5, it can be concluded that using sorted coefficients, better imperceptibility can be achieved. Another important thing observed in Figure 2- Figure 5 is that column transform gives the highest imperceptibility among all. In the following sections we focus on robustness of proposed method by evaluating its performance against various attacks. Various attacks performed on watermarked images are cropping, compression, noise addition, histogram equalization and resizing. These attacks except histogram equalization are performed in variety of ways. For e.g. compression attack is performed using various transforms like DCT, DST, Walsh, Haar, JPEG compression and Vector quantization based compression.

\subsubsection{Results of cropping attack:}

Figure 6 shows results of cropping a watermarked image at four corners (16x16) using full Haar transform and column and row version of Haar transform.



Figure 6: Watermarked image and recovered watermark from it when 16x16 cropping is performed with and without sorting transform coefficients: (a) Full transform HL band (b) Full transform LH band (c) Column transform (d) Row transform 
H. B. Kekre, Tanuja Sarode and Shachi Natu; Performance Improvement by Sorting the Transform Coefficients of Host and Watermark using Unitary Orthogonal Transforms Haar, Walsh and DCT, Advances in Image and Video Processing, Volume 2 No 4, Aug (2014); pp: 24-38

Figure 6 shows that in sorting method, HL band when used for embedding the watermark gives minimum MAE between embedded and recovered watermark. This error is much less than the error when transform coefficients are not sorted. For LH band used for watermark embedding also similar effect is observed. However, the trend of reduction in error does not follow for column and row transform.

\subsubsection{Compression attack:}

Compression attack using various orthogonal transforms, using vector quantization and JPEG compression is performed on watermarked images. Results of DCT based compression, JPEG compression and VQ based compression with and without sorting technique are shown in Figure 7 to Figure 9 respectively.



Figure 7: Watermarked image and recovered watermark from it when compression using DCT is performed with and without sorting transform coefficients: (a) Full transform HL band (b) Full transform LH band (c) Column transform (d) Row transform

From Figure 7, it is observed that sorting the transform coefficients for embedding reduces the MAE between embedded and recovered watermark. Among them, Column version of transform is more robust followed by row transform than other versions (full HL and Full LH).

Figure 8 shows watermarked images after JPEG compression and recovered watermark from it. Column Haar transform proves to be more robust for JPEG compression attack and column as well as row transform with sorted coefficients give better imperceptibility and robustness than in case of unsorted elements. However, full transform with sorted transform coefficients does not give better robustness than full transform with unsorted coefficients.

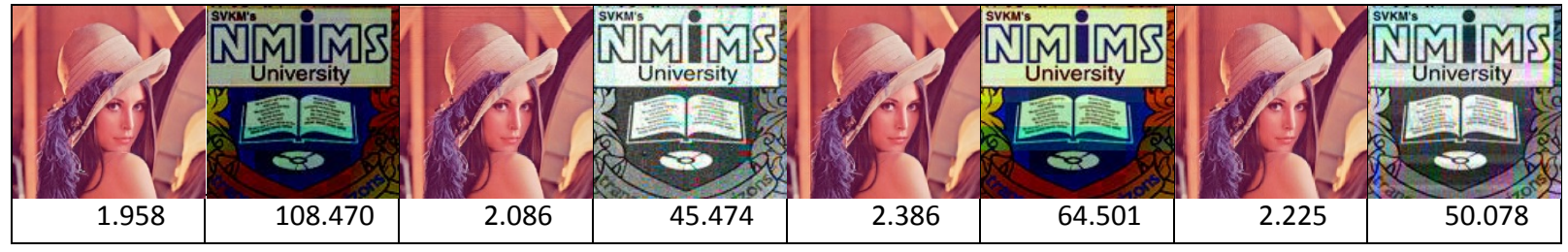






Figure 8: Watermarked image and recovered watermark from it when JPEG compression is performed with and without sorting transform coefficients: (a) Full transform HL band (b) Full transform LH band (c) Column transform (d) Row transform

Figure 9 shows watermarked images compressed using Vector Quantization performed using KFCG algorithm [19] with codebook size 256. Full transform with sorted coefficients give less distorted image than in case of unsorted coefficients but due to color changes it shows high MAE between embedded and recovered watermark. Recovered watermark quality is better in column and row Haar transform using sorted coefficients and row transform shows minimum MAE value i.e. strong robustness among all.

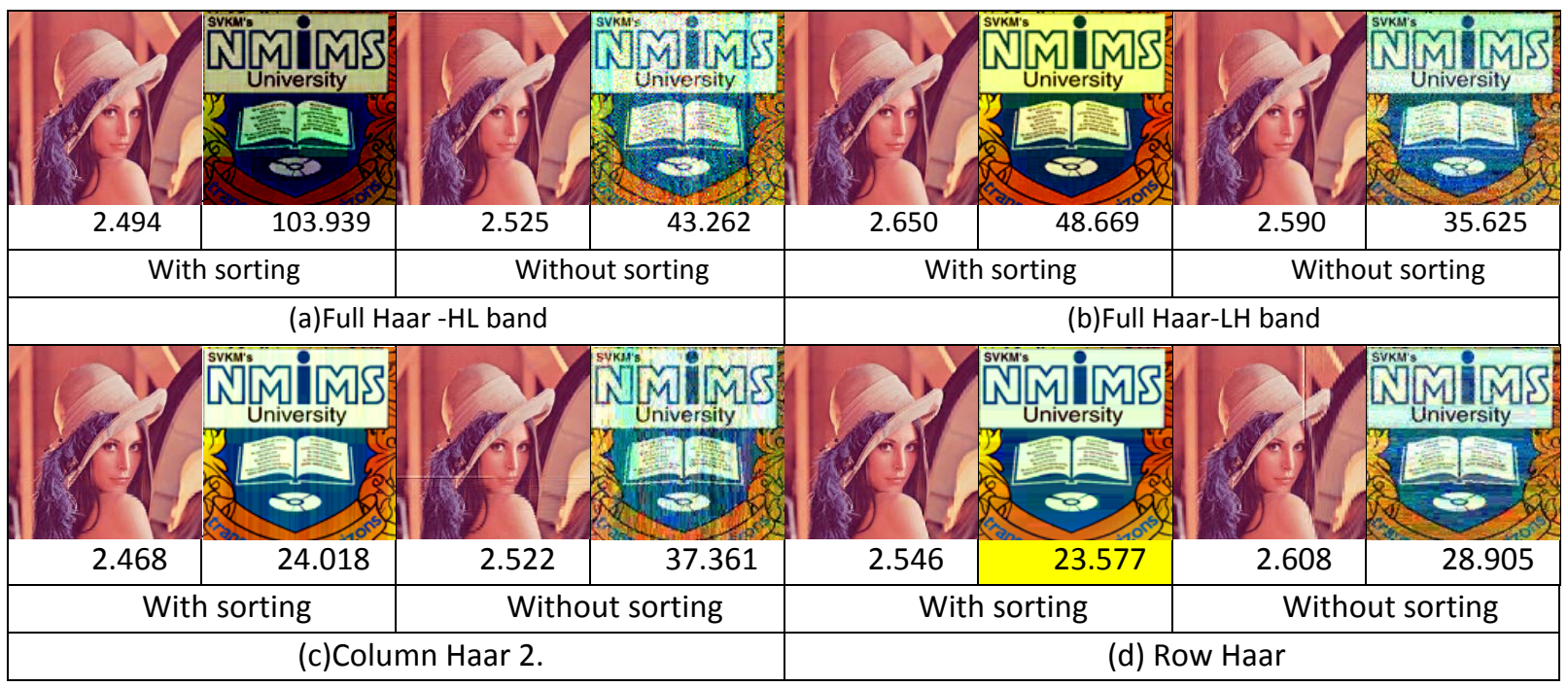

Figure 9: Watermarked image and recovered watermark from it when VQ compression is performed with and without sorting transform coefficients: (a) Full transform HL band (b) Full transform LH band (c) Column transform (d) Row transform

\subsubsection{Noise addition attack:}

Addition of noise to image during its transmission is yet another common attack possible after compression. In the proposed method binary distributed run length noise and Gaussian distributed run length noise are introduced and are added to watermarked image. Binary distributed noise has magnitude 0 and 1 with normal distribution. Different run lengths of binary distributed noise are tried like run length 1 to 10,5 to 50 and 10 to 100 . Gaussian distributed run length noise has discrete magnitude between -2 and 2 . Figure 10 shows noise added watermarked images with binary run length noise (run length 10 to 100) and recovered watermarks. From Figure 10 it can be seen that sorting gives better robustness in all cases. 
H. B. Kekre, Tanuja Sarode and Shachi Natu; Performance Improvement by Sorting the Transform Coefficients of Host and Watermark using Unitary Orthogonal Transforms Haar, Walsh and DCT, Advances in Image and Video Processing, Volume 2 No 4, Aug (2014); pp: 24-38

Among them full transform with LH band gives zero error followed by row transform, full transform $\mathrm{HL}$ band and column transform.

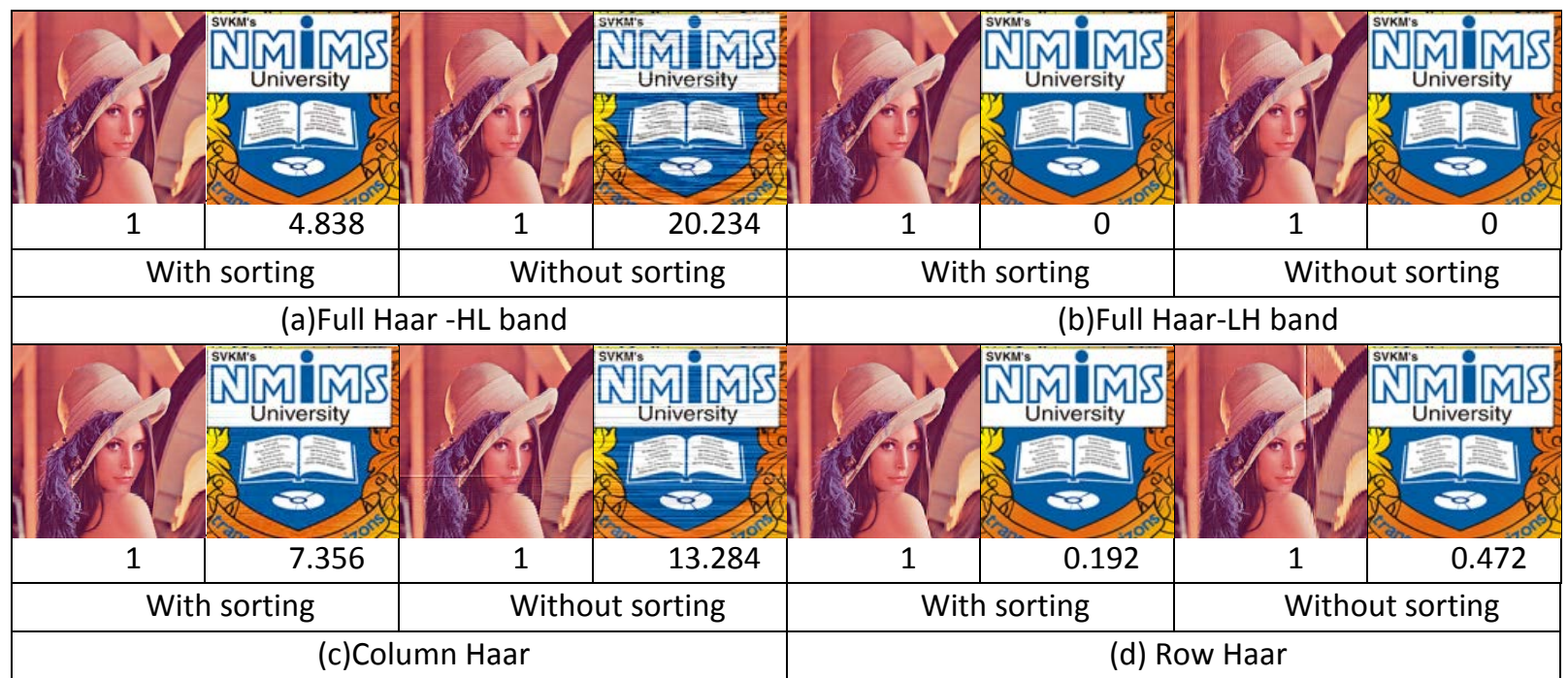

Figure 10: Watermarked image and recovered watermark from it when binary distributed run length noise is added with and without sorting transform coefficients: (a) Full transform HL band (b) Full transform LH band (c) Column transform (d) Row

transform

Figure 11 shows watermarked images when Gaussian distributed run length noise is added to it and recovered watermark from it. It is observed that sorting coefficients in $\mathrm{HL}$ band gives best robustness among all. It is also equal to the performance of embedding in unsorted coefficients. For LH band of full transform as well as row and column transform, robustness improves when transform coefficients are sorted.

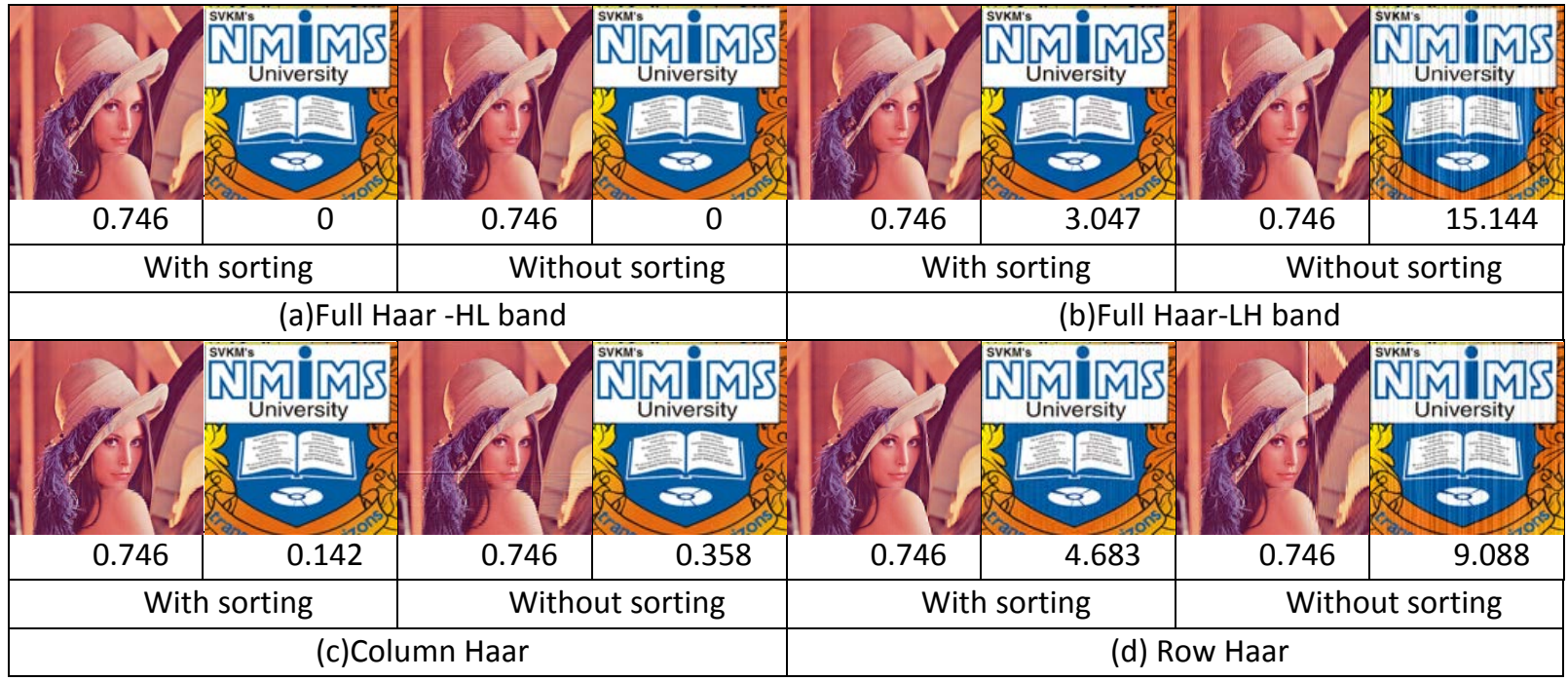

Figure 11: Watermarked image and recovered watermark from it when Gaussian distributed run length noise is added with and without sorting transform coefficients: (a) Full transform HL band (b) Full transform LH band (c) Column transform (d)

Row transform 


\subsubsection{Image resizing attack:}

In resizing attack watermarked image is zoomed to double its size and then resized to its original size using techniques like bicubic interpolation, grid based interpolation [20] and orthogonal transform based zooming [21]. Figure 12 shows result images for zoomed-reduced watermarked images using bicubic interpolation and recovered watermarks from them for full, column and row Haar transform. As can be seen from Figure 12, in each case (full, column and row) sorting of transform coefficients results in better robustness. Row Haar transform shows better robustness among all when sorting is used.

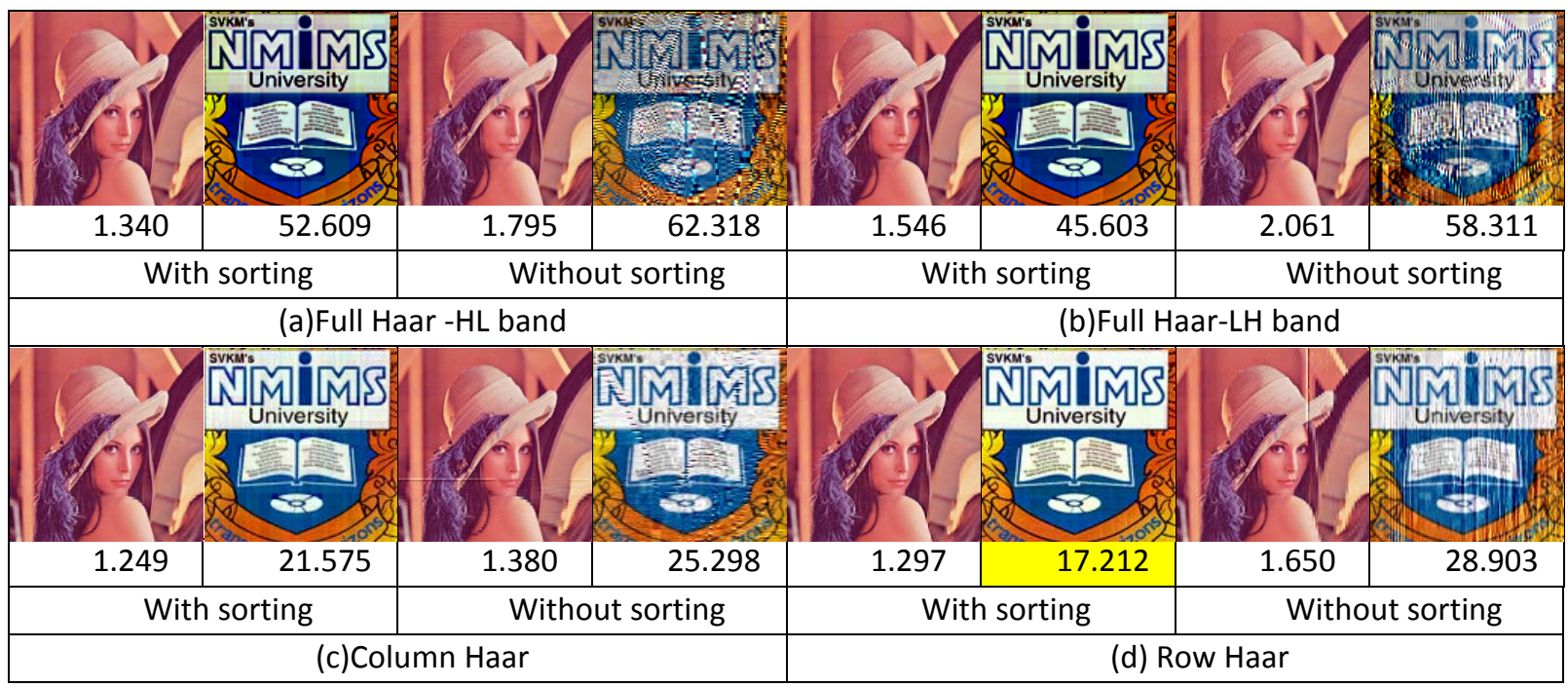

Figure 12: Zoomed-reduced Watermarked image and recovered watermark from it using bicubic interpolation with and without sorting transform coefficients: (a) Full transform HL band (b) Full transform LH band (c) Column transform (d) Row transform

Figure 13 shows resizing attack performed using grid based interpolation technique and recovered watermarks from it. Column Haar transform with sorted transform coefficients gives more robustness than full and row transform. Full transform using $\mathrm{LH}$ band for embedding the watermark also shows reduced MAE between embedded and recovered watermark. But full transform using $\mathrm{HL}$ band for embedding watermark fails to give robustness as it shows strong color manipulation taking place in recovered watermark.




H. B. Kekre, Tanuja Sarode and Shachi Natu; Performance Improvement by Sorting the Transform Coefficients of Host and Watermark using Unitary Orthogonal Transforms Haar, Walsh and DCT, Advances in Image and Video Processing, Volume 2 No 4, Aug (2014); pp: 24-38

\begin{tabular}{|c|c|c|c|}
\hline With sorting & Without sorting & With sorting & Without sorting \\
\hline \multicolumn{2}{|c|}{ (c)Column Haar } & (d) Row Haar \\
\hline
\end{tabular}

Figure 13: Zoomed-reduced Watermarked image and recovered watermark from it using grid based interpolation with and without sorting transform coefficients: (a) Full transform HL band (b) Full transform LH band (c) Column transform (d) Row transform

Figure 14 shows resized watermarked images using Discrete Fourier transform and recovered image from them. For full transform $\mathrm{HL}$ and $\mathrm{LH}$ band sorting of transform coefficients improves robustness as well as imperceptibility. For column transform robustness almost remains same in both the cases and for row transform again it shows minor improvement in robustness.

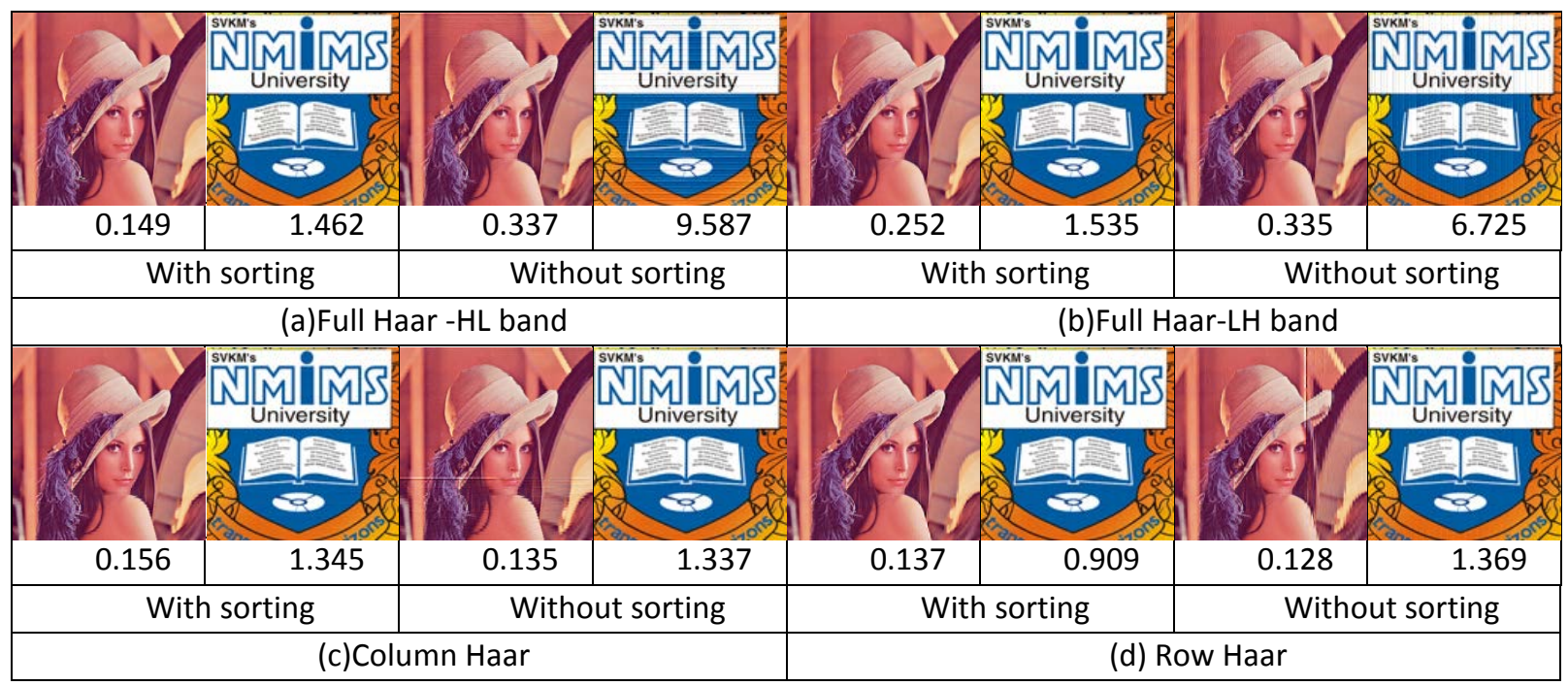

Figure 14: Zoomed-reduced Watermarked image and recovered watermark from it using DFT based zooming with and without sorting transform coefficients: (a) Full transform HL band (b) Full transform LH band (c) Column transform (d) Row transform

\subsection{Performance comparison of proposed technique with technique using unsorted transform coefficients for various attacks.}

In Figure 15, graph of comparison of full Haar transform HL band with and without sorting and $100 \%$ embedding energy is shown for various attacks performed on watermarked Lena image. Performance of proposed sorting technique is better for every attack performed on watermarked image prominently except for resizing using grid based interpolation, VQ based compression and JPEG compression and to very small extent for 32x32 cropping at center. 




Figure 15: Performance comparison of full Haar transform HL band with embedding energy $100 \%$ with and without using sorting for 'Lena' image

Figure 16 shows graph of MAE comparison when full Haar transform is taken and LH band is selected to embed the watermark after sorting transform coefficients. For LH band also sorting results in better robustness. An improvement seen in LH band embedding is that for grid based resizing method sorting technique gives better robustness than without sorting the transform coefficients. For VQ based compression and JPEG compression, sorting does not prove effective than unsorted coefficients.

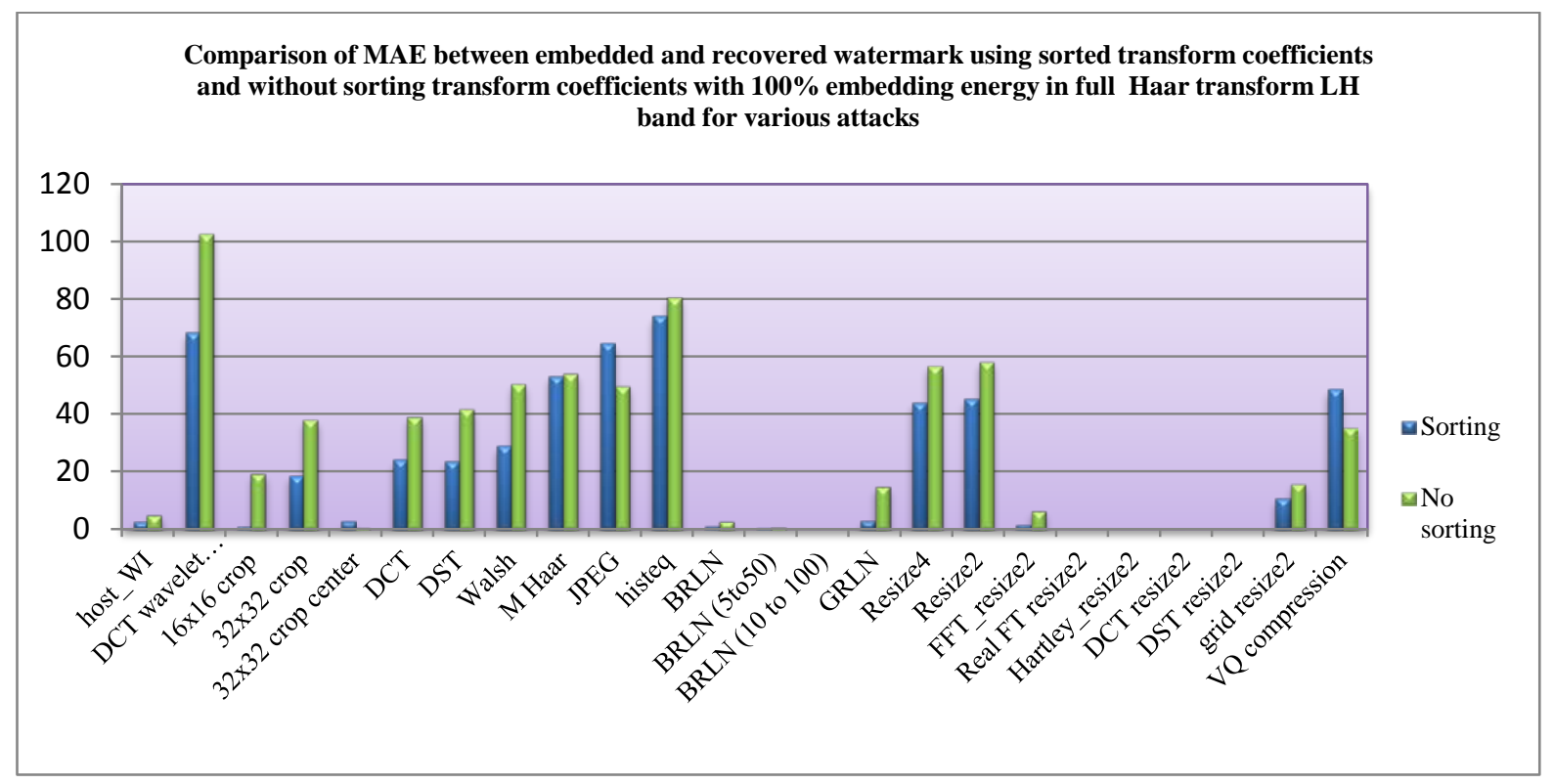

Figure 16: Performance comparison of full Haar transform LH band with embedding energy $100 \%$ with and without using sorting for 'Lena' image

Graphs in Figure 17 and Figure 18 show comparison of MAE between embedded and recovered watermark for various attacks when column and row Haar transforms are used 
H. B. Kekre, Tanuja Sarode and Shachi Natu; Performance Improvement by Sorting the Transform Coefficients of Host and Watermark using Unitary Orthogonal Transforms Haar, Walsh and DCT, Advances in Image and Video Processing, Volume 2 No 4, Aug (2014); pp: 24-38

respectively both with and without sorting of transform coefficients and $100 \%$ embedding energy. Both column and row transform show significant improvement in robustness with sorting over $\mathrm{HL}$ and $\mathrm{LH}$ band of full transform for JPEG compression, VQ based compression and grid based resizing attack. For other attacks also it gives better robustness than unsorted transform coefficients.

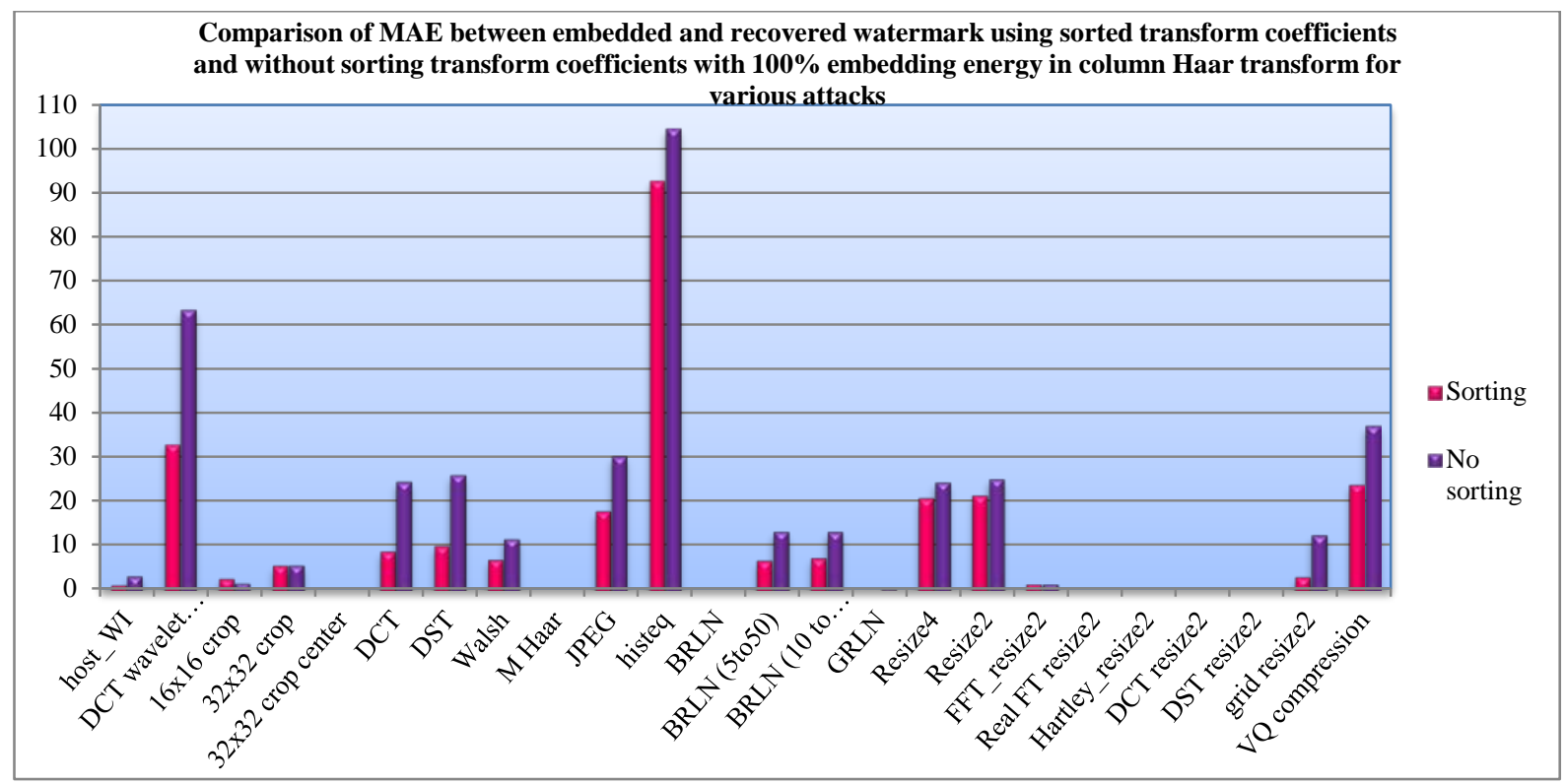

Figure 17: Performance comparison of column Haar transform with embedding energy $100 \%$ with and without using sorting for 'Lena' image

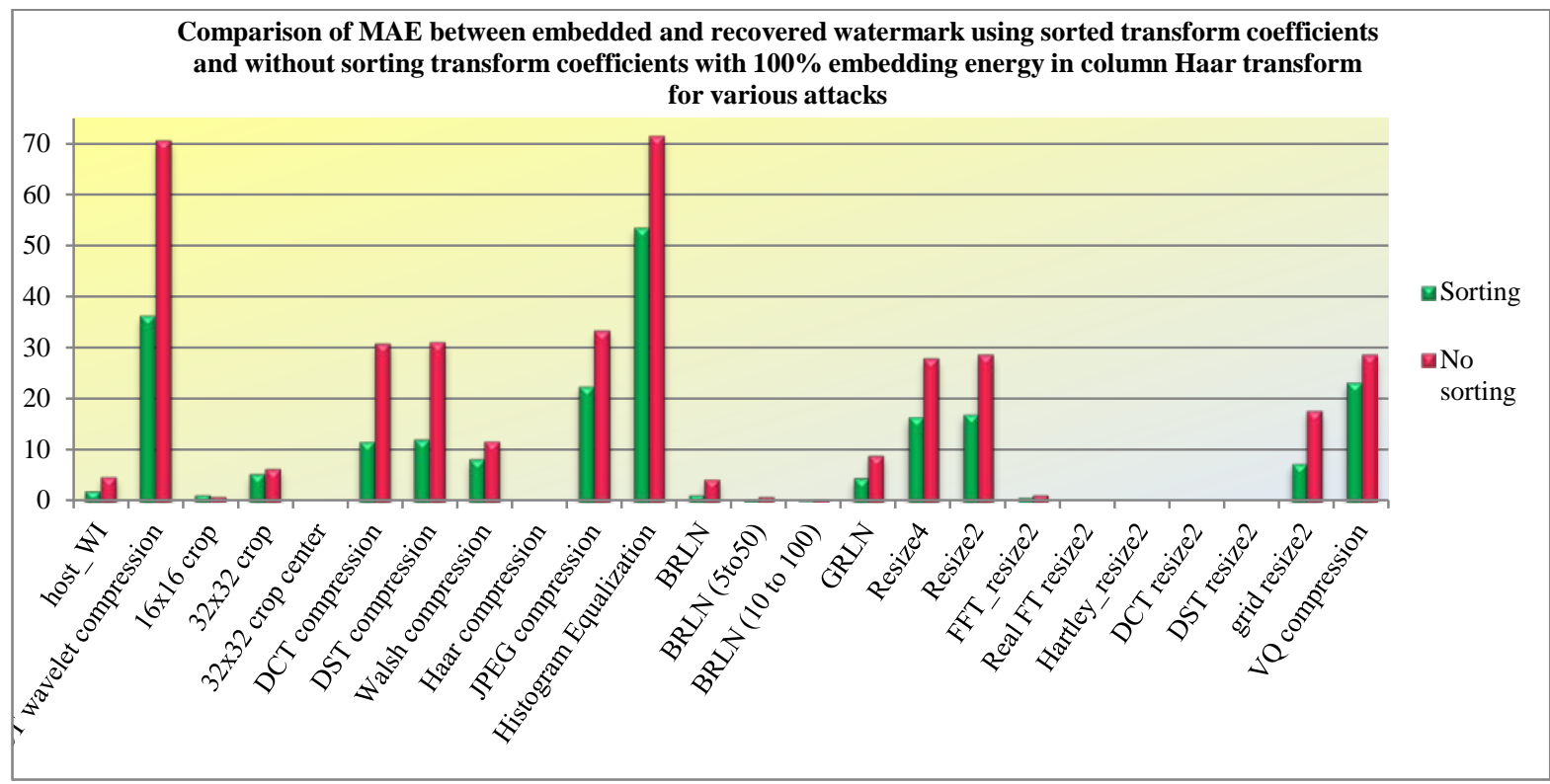

Figure 18: Performance comparison of row Haar transform with embedding energy $100 \%$ with and without using sorting for 'Lena' image

\section{Conclusion}


Sorting of transform coefficients of watermark and host minimizes the error between corresponding elements and hence overall error of embedding the watermark. Full Haar $\mathrm{HL}$ band results in better robustness than in case where transform coefficients are not sorted except JPEG and VQ compression attack and grid based resizing. Full Haar LH does better by eliminating grid base resizing from the above list. Column and row Haar transform are proved better in terms of robustness than full transform ( $\mathrm{HL}$ and $\mathrm{LH}$ band). This is true for other transforms explored i.e. Walsh and DCT too.

\section{REFERENCES}

[1]. Houtan Hadded Larijani, Gholamali Rezai Rad, "A new spatial domain algorithm for gray scale images watermarking", In proc. of international conference on computer and communication engineering, pp. 157161, May 2008.

[2]. Rajesh Kannan Megalingam, Mithun Muralidharan Nair, Rahul Srikumar, Venkat Krishnan Balasubramanian, Vineeth Sarma Venugopala Sarma, Chapter titled, "Novel Variance based Spatial Domain Watermarking and its Comparison with DIMA and DCT based Watermarking Counterparts", in the book, "Applications of MATLAB in Science and Engineering", ISBN 978-953-307-708-6, Intech Open Access Publisher, 2011, ch. 22, pp. 451-468

[3]. Ibrahim Nasir, Ying Weng, Jianmin Jiang, "A new robust watermarking scheme for color image in spatial domain", In proc. of third international conference on signal image technologies and internet based systems, pp. 942-946, 2008.

[4]. Saba Riaz, Yonus Javed, M. Almas Anjum, "Invisible watermarking schemes in spatial and frequency domains", In proc. of 4th international conference on emerging technologies, pp. 211-216, 2008.

[5]. W. Lu, H Lu and F. L. Chung, "Robust digital image watermarking based on subsampling", Applied mathematics and computation, vol. 181, pp.886-893, 2006

[6]. V. I. Licks, "On digital image watermarking robust to geometric transformations", Master of science Electrical engineering, university of New Mexico, 2000.

[7]. I. Cox, J. Killian, T. Leighton and T. Shamoon, "Secure spread spectrum watermarking for multimedia", IEEE transaction on image processing, 6(12), 1673-1687, December 1997.

[8]. C. S. Woo, J. Du, B. Pham, "Performance factor analysis of a wavelet based watermarking methods", in Proc. of Australian information security workshop, vol. 44, pp. 89-97, Feb. 2005.

[9]. A. Giakoumaki, S. Pavlopoulo, D. Koutouris, "A medical image watermarking scheme based on wavelet transforms", In proc. of $25^{\text {th }}$ annual international conference of the engineering in medicine and biology society, IEEE, vol. 1, pp.856-859, September 2003.

[10]. H. B. Kekre, Tanuja Sarode, Shachi Natu, "Performance Comparison of DCT and Walsh Transforms for Watermarking using DWT-SVD", International Journal of Advanced Computer Science and Applications, Vol. 4, No. 2, pp. 131-141, 2013. 
H. B. Kekre, Tanuja Sarode and Shachi Natu; Performance Improvement by Sorting the Transform Coefficients of Host and Watermark using Unitary Orthogonal Transforms Haar, Walsh and DCT, Advances in Image and Video

Processing, Volume 2 No 4, Aug (2014); pp: 24-38

[11]. Dr. H. B. Kekre, Dr. Tanuja Sarode, Shachi Natu, "Hybrid Watermarking of Colour Images using DCT-Wavelet, DCT and SVD", International Journal of Advances in Engineering and Technology, vol.6, Issue 2. May 2013.

[12]. Dr. H. B. Kekre, Dr. Tanuja Sarode, Shachi Natu, "Robust watermarking using Walsh wavelets and SVD", International Journal of Advances in Science and Technology, Vol. 6, No. 4, May 2013.

[13]. Dr. H. B. Kekre, Dr. Tanuja Sarode, Shachi Natu," Performance Comparison of Wavelets Generated from Four Different Orthogonal Transforms for Watermarking With Various Attacks", International Journal of Computer and Technology, Vol. 9, No. 3, pp. 1139-1152, July 2013.

[14]. Dr. H. B. Kekre, Dr. Tanuja Sarode, Shachi Natu, "Performance of watermarking system using wavelet column transform under various attacks", International Journal of Computer Science and Information Security, Vol. 12, No. 2, pp. 30-35, Feb 2014.

[15]. Dr. H. B. Kekre, Dr. Tanuja Sarode, Shachi Natu, "Robust watermarking scheme using column DCT wavelet transform under various attacks", International Journal on Computer Science and Engineering, Vol. 6, No. 1, pp. 31-41, Jan 2014.

[16]. Dr. H. B. Kekre, Dr. Tanuja Sarode, Shachi Natu, "Performance evaluation of watermarking technique using full, column and row DCT wavelet transform", International Journal of Advanced Research in Electrical, Electronics and Instrumentation Engineering, Vol. 3, Issue 1, Jan 2014.

[17]. Dr. H. B. Kekre, Dr. Tanuja Sarode, Shachi Natu, "Robust watermarking technique using hybrid wavelet transform generated from Kekre transform and DCT", International Journal of Scientific Research Publication, vol. 4, Issue 2, pp. 1-13, Feb 2014.

[18]. Dr. H. B. Kekre, Dr. Tanuja Sarode, Shachi Natu, "Effect of weight factor on the performance of hybrid column wavelet transform used for watermarking under various attacks", International Journal of Computer and Technology, Vol. 12, No. 10, pp. 3997-4013, March 2014.

[19]. H. B. Kekre, Archana A. Athawale, Dipali Sadavarti, “ Algorithm to Generate Kekre's Wavelet Transform from Kekre's Transform”, International Journal of Engineering, Science and Technology, (IJEST) Vol. 2(5), 756-767, 2010.

[20]. H. B. Kekre, Tanuja Sarode, Sudeep Thepade, “Grid based image scaling technique”, International Journal of Computer Science and Applications, Volume 1, No. 2, pp. 95-98, August 2008.

[21]. Dr. H. B. Kekre, Dr. Tanuja Sarode, Shachi Natu, “Image Zooming using Sinusoidal Transforms like Hartley, DFT, DCT, DST and Real Fourier Transform", selected for publication in International journal of computer science and information security Vol. 12 No. 7, July 2014. 